\title{
Original
}

\section{Analysis of 200 cases with Pediatric Anorectal Malformations}

Norma Ceciliano-Romero, Debora Beauchamp-Carvajal, Laura Chavez-Fernández, Jaime Cortés-Ojeda

Surgery Department, Hospital Nacional de Niños Dr. Carlos Sáenz Herrera

Contact information:

E-mail: nocerodd@hotmail.com

\section{Abstract}

Aim: Anorectal malformations are a frequent pathology in the general surgery service at the National Children's Hospital, however, in Costa Rica, there have been few publications about this topic. The objective of this work is to analyze patients that were operated between 1998 and 2008 , to know in detail the treatments used and to determine what has the experience been, this is of extreme importance, according to the findings, to process suggestions to make improvements.

Methods: Clinical files were reviewed and all the patients with anorectal malformations operated between 1990 and 2008 were examined, controlled at the outpatient service of General Surgery at the National Children's Hospital. The patients were grouped according to their type of anomaly and were examined to determine: congenital malformations, type of anorectal malformations, type of surgery, complications and results from the following tests: abdominal ultrasound, voiding cystourethrography, distal colography and its functional results, after three years of age. The patient's data were placed in a data base under the Excel program to be able to analyze them and have a clear vision of the procedures utilized with each group and the results obtained.

Results: 200 cases operated between 1998 and 2008 were controlled; all were studied for congenital malformations and anal malformations. Different groups were found which were classified the following manner: those who had fistulas which were subdivided by the location of the opening: bladder neck (12 cases), prostatic urethra (12 cases), bulbar urethra (18 cases), vestibular (26 cases), perineum (92 cases), anal stenosis (12 cases); those who had no fistula: high (16 cases) or low (3 cases), and finally, cloacal malformations: high ( 2 cases), and low ( 7 cases). All the patients underwent surgery using the Peña 11 technique with the corresponding modifications. No patient was intervened utilizing laparoscopic technique. 
Conclusion: These patients are very complex and must be analyzed as a whole since their malformations are very important in the results and morbid-mortality. In most cases good results were obtained. The important aspect is to control them for a long period of time and use the procedures necessary to allow a normal life style, which is possible.

Keywords: anorectal malformation, cloacal, colostomy, fistula, laparoscopy, colography.

Received: $24^{\text {th }}$ September $2009 \quad$ Accepted: $9^{\text {th }}$ February2009

Children born with an anorectal malformation are complex patients, in occasions with multiple malformations. It is fundamental to diagnose precisely all the anomalies as soon as possible to offer an appropriate treatment.

Associated malformations are of great importance, they are the ones that produce mortality since the anal lesions that cause intestinal obstruction are correctable with surgery. Only when they are not operated or are operated later on, will they produce mortality.

Since there are several types of anorectal malformations, it is important to classify and group the patients in the corresponding type. The classification used divides them in those with fistula and those without. The first are subdivided according to the location of the opening, the latter are subdivided into upper and lower. Cloacas are studied in a separate group defined as the junction of the rectus, the vagina and urethra. There are two types of cloacas: the high ones where the common channel is more than $3 \mathrm{~cm}$ and the low ones where the channel measures less than $3 \mathrm{~cm}$. This classification is necessary since the treatments and results are different.

The correct diagnosis of the lesions should follow certain guidelines which vary from clinical observation of perineum area, which allows an $8 \%$ diagnosis of the cases by determining the existence of the perineum and vestibular fistulas and a single orifice when referring to the cloacal fistulas. In the rest of the patients, contamination of urine with meconium clears the diagnosis of a fistula. When there is absence of this contamination, the fistula is discarded and the diagnosis is done by a simple radiography, taken in a transversal manner, with a roller that presses over the abdomen and a metal mark placed were the anus should be. If the distance between the area image and the mark is greater than $1 \mathrm{~cm}$ it is classified as high, and if it is less, it is classified as a low. This test must be done after 24 hours of birth in order to wait until the air arrives to the rectal pouch.

In those cases where patients with fistulas have undergone a colostomy, a distal colography with pressure is the test which shows where the opening is. In order to be able to see the fistula, requirements must be met: a) have a clean distal segment of the colostomy and b) place pressure when introducing the contrast medium. If these requirements are not accomplished, a high percentage of fistulas will not be diagnosed.

$\mathrm{T}$ he objective of this paper is to analyze the patients with anorectal malformations operated between 1998 and 2008, who arrived to the surgery consult at the Dr. Carlos Saenz Herrera National Children's Hospital. 


\section{Methods}

The control was made by revised clinical files and the patient's tests. This is a retrospective study, descriptive, in order to know the details of treatments used and to determine what the experience has been, with the purpose of proposing suggestions which allow for better results.

The patients were classified according to the following criteria: if they had a fistula or not, and in case they did have one, they were grouped according to the location of the opening: bladder neck, prostatic urethra, bulbar urethra, vestibular, perineum and anal stenosis. Those patients who did not have fistulas were divided into high and low; another group was made up of cloacas, which could also be high or low.

Two diagnostic criteria were analyzed: one was to group them up according to the type of malformation; where the clinic, study of simple transversal radiography, mark in the perineum, and distal colography, were fundamental. The other, to diagnose the congenital malformations based on the following tests: abdominal ultrasound, voiding urethrography and neurological and cardiac evaluation.

Special attention was granted to the different surgical procedures performed, the complications that arose and the results obtained. The latter criteria was considered normal for those who passed stools regularly and did not need any treatment; constipated to those who required some treatment to obtain a normal stool rate; fecally incontinent to the patient that does not have voluntary control of his stool and that may have an increased or decreased intestinal motility, for which they may be constipated or Acta méd. costarric. Vol 52 (2), April- June 2010 have diarrhea, and pseudoincontinent to those who soil because they do not pass stool and instead accumulate it in a dilated rectus.

In most of the patients, the sacral index (SI) was measured, in the same manner as it was described by Peña, for its importance in the result's prognosis. The data obtained from the clinical files were introduced into an Excel data base and analyzed using a dynamic table, which allowed a detailed knowledge of the different types of malformations, which were analyzed separately to be able to compare the results.

Associated congenital malformations were analyzed as a whole, including all the patients however, each group mentions the data of the abdominal ultrasound and the voiding cystourethrography (VCUG) in search of determining the specific malformations.

\section{Results}

Two hundred patients were analyzed with anorectal malformations, all of them treated at the Dr. Carlos Saenz Herrera Children's National Hospital, between 1998 and 2008, which were controlled in the outpatient service of General Surgery.

They were organized according to the classification shown in Figure $1^{1}$ : those who had a fistula located at the opening: bladder neck (12 cases), prostatic (12 cases), bulbar (18 cases), vestibular (26 cases), perineum (92 cases), anal stenosis (12 cases), those without fistulas: high (16 cases), or low (3 cases), and finally the cloacal malformations, high ( 2 cases) and low ( 7 cases). 
All the patients were operated using the Peña technique, ${ }^{2}$ with the corresponding modifications. No patient was intervened using the laparoscopic technique, but reference is made in the commentary, for its indication.

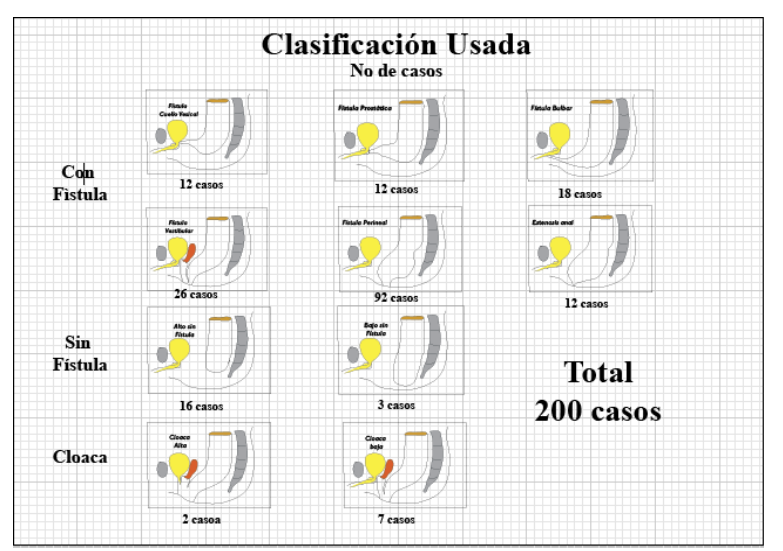

Figure 1

All patients were operated by surgeons at the General Surgery Service at the National Children's hospital for their anorectal lesions. The corresponding malformations were resolved by the adequate specialist.

\section{Bladder neck fistula}

There were 12 patients diagnosed under this classification, all had a flat perineum and urine contamination with feces. Distal colography showed a fistula towards the bladder in 6 cases, and in one of them, there was an opening towards the prostatic urethra. The remaining 5 cases had no data that indicated a fistula.

The abdominal US showed: 6 normal patients, 3 with hydronephrosis, 2 with solitary kidneys and 1 without data. The results of the VCUG were as follows: 1 with urethral stricture, 1 with an ureterocolic fistula (ectopic ureter), 3 megacystis, 1 postvoiding residue, 1 vesicoureteral reflux (VUR) and 5 normal cases.

Acta méd. costarric. Vol 52 (2), April- June 2010
Two children had an associated esophageal atresia, which is corrected with a colostomy.

Surgeries practiced on these patients with different types of anorectal malformations are shown in Table 1.

All 12 patients were colostomized, a PSARP + abdominal time was performed in 12 of them, and in one case, the colostomy has not closed yet.

Complications included: 1 patient had an inverted colostomy, which evolved into an ileal perforation, this is the same case in which the colostomy has not closed yet, therefore it has not been evaluated. Table 2 shows the patient's complications with different types of anorectal malformations.

The functional results were: 10 incontinent, 3 had sacral index less than 0.3 and 1 had sacral agenesis, ${ }^{3}$ as shown in Table 3.

\section{Prostatic fistula}

There were 12 patients with this type of fistula; all of them presented contamination of the urine by meconium and a flat perineum. Distal colography showed the fistula in 9 cases, 1 was reported as a bulbar fistula. 2 cases did not show the fistula. The abdominal US showed: 1 solitary kidney, 1 horseshoe kidney, 9 normal and one without data. The VCUG demonstrated one case of an ectopic kidney, 2 prostatic fistulas, 1 hydronephrosis, 1 bladder stones, 1 solitary kidney, 1 case of postvoiding residue, 4 normal and 1 without data. The 12 surgeries performed were colostomies followed by PSARP and closure of colostomies in a tertiary surgery.

Complications were as follows: 2 inverted colostomies where there was no need to reoperate, and 2 urethral ruptures that resolved during de PSARP, 1 patient had dehiscence of the anal suture and was re-intervened. The results 
were: 5 incontinent, 2 constipated, 3 continent and 2 which had not been evaluated.

\section{Bulbar fistula}

The 18 cases in this group had contamination of the urine with meconium and a fistula was able to be documented in 7 of them with a distal colography, 6 were reported without a fistula and 5 had no data in the clinical file.

The abdominal US documented: 2 cases with right renal atrophy, 1 case with horseshoe kidney, 1 solitary kidney and 11 normal cases; 4 cases had no data.

The VCUG documented: 2 bulbar fistulas, 4 VUR, 9 normal and 3 had no data. The surgical results were: 18 cases of colostomy followed by PSARP and closure of the colostomy. The results were as follows: 5 incontinent, 5 constipated, 4 continent, and 4 have not yet been classified.

\section{Vestibular fistula}

Out of the 26 patients who make up this group, all were diagnosed by exploring the perineum, only 2 of them using a distal colography, despite that this test is not necessary to diagnose a fistula. The abdominal US documented: 1 pyelocalyceal ectasia, 2 solitary kidneys, 1 normal medulla, 21 normal and one without data. The VCUG documented: 1 case with hydroneprhosis, 2 megacystis, 1 solitary kidney, 3 VUR, 1 with a stress bladder, 1 with residual postvoiding, 15 normal, and 2 without data. Surgeries performed were: colostomy (12 cases), PSARP (26 cases), closure of colostomy (21 cases), reoperation of PSARP (1 case). The only complication was 1 dehiscence of the anal suture. The results were the following: 3 incontinent, 1 with sacral agenesis and another with SI less than $0.3,5$ constipated, 1 pseudoincontinent, 12 continent, 4 without evaluation.

Acta méd. costarric. Vol 52 (2), April- June 2010

\section{Perineum fistula}

This group was made up of 92 patients diagnosed by exploration of the perineum. The abdominal US documented: 2 pyelocalyceal ectasias, 3 hydronephrosis, 5 solitary kidneys, 2 horseshoe kidneys, 2 with normal medulla, 57 normal and 20 without data. VCUG showed: 1 Hutch diverticulum, 1 hydronephrosis, 1 megacystis, 5 VUR, 1 posterior ureteral valve, 2 stress bladders, 60 normal and 21 without data. The surgeries performed were: colostomies (7 cases), limited PSARP (83 cases), closure of colostomies ( 7 cases), reoperations of PSARP (11 cases), dilations ( 8 cases.) One patient had and esophageal atresia that was corrected by performing a colostomy. In the case of 4 patients who underwent colostomy, this obeyed a late diagnosis. The complications were as follows: 11 patients with dehiscence of the anal suture, which had to undergo surgery again. One patient developed a megasigmoid which was removed when the closure of the colostomy was performed. The results were: 6 incontinent, 20 constipated, 2 pseudoincontinent, 27 continent and 27 have not yet been evaluated.

\section{Anal stenosis}

The 12 cases in this group were diagnosed by exploring the perineum area. No patient underwent a distal colography. The abdominal US documented: 8 normal and 4 had no data. The VCUG documented: 1 postvoidal residue, 1 VUR, 1 neurogenic bladder, 7 normal and 2 without data. Surgeries performed were: colostomy, PSARP and colostomy closure (1 case), radiated incisions (2 cases), dilations (6 cases), and sigmoidectomy (1 case). There were no complications. Results were as follows: 4 constipated, 1 psuedoincontinent, 5 continent and 2 where not evaluated.

\section{High without fistula}


The 16 patients in this group were diagnosed by the perineum exploration and the distance between the rectum and the mark in the perineum was always greater than $1 \mathrm{~cm}$. Distal colography documented: 11 without fistula, 1 fistula at the bladder neck and 4 had no data. The abdominal US documented: 2 pyelocalyceal ectasia, 1 solitary kidney, 10 normal, 2 without fistula and 1 without data.

VCUG documented: 1 megacystis, 2 VUR, 10 normal, 1 with bladder stricture and 2 without data. Surgeries performed were: colostomies (15 cases) PSARP (16 cases), closure of colostomies (15 cases) and reintervention of PSARP ( 1 case). Complications were as follows: 2 urethral ruptures, 1 bladder diverticulum, 1 acute appendicitis. Results included: 1 incontinent, 6 constipated, 7 continent, 2 without evaluation. One of the patients of this group had an esophageal atresia which was corrected when colostomy was performed.

The patient who underwent PSARP as primary surgery is one of the 2 who had rupture of the urethra.

\section{Low without fistula}

The 3 patients in this group were diagnosed by exploring the perineum and by a simple transversal radiography using a roll on the abdomen. The distance in all three, between the air in the rectus and the mark in the perineum, was lower than $1 \mathrm{~cm}$. Only one case had a colostomy performed and distal colography demonstrated the fornix of the rectus without fistula. The abdominal US documented: 1 solitary kidney, and 1 normal, 1 without fistula. The VCUG documented: 1 megacystis, 1 VUR, and 1 without data. 2 cases underwent a limited PSARP as the only surgery, and one had a colostomy, with a limited PSARP during a secondary operation and closure of colostomy during the tertiary operation. There was replacement of a colostomy as a complication.

Acta méd. costarric. Vol 52 (2), April- June 2010
The results included: 1 continent case and 2 constipated cases.

\section{High cloaca}

The 2 patients in this group were diagnosed by exploring the perineum. Distal colography did not show data in any of the patients. The abdominal US documented: 1 high cloaca and 1 without data. VCUG documented both cases without data. A colostomy and cystostomy were performed in one case and a colostomy and vaginostomy in the other in the first surgery, PARUVP in the secondary one and closure of colostomy in the tertiary operation.

There were no complications. Both patients are incontinent.

\section{Low cloaca}

The 7 patients in this group were diagnosed by exploring the perineum. Distal colography documented: 1 low cloaca, 4 vestibular fistulas, and 2 without data. The abdominal US documented: 1 pyelocalyceal ectasia, 1 hydronephrosis, 4 normal and 1 without data. VCUG showed: 1 case with contrast medium in the tube, 1 vaginal fistula, 3 normal, 1 VUR and 1 without data.

All 7 cases were managed with a colostomy as first surgery. In one of them, the esophageal atresia was corrected at the same time. 5 patients underwent PARUVP as a secondary surgery, with a fall of the sinus and 2 underwent PARUVP only. All had closure of colostomy performed at the tertiary surgery. Complications included: 2 patients with trouble at the anal suture, who had to be reoperated, the other 5 had no complications. The functional results were: 2 continent, 2 constipated, 2 incontinent and 1 without data. 


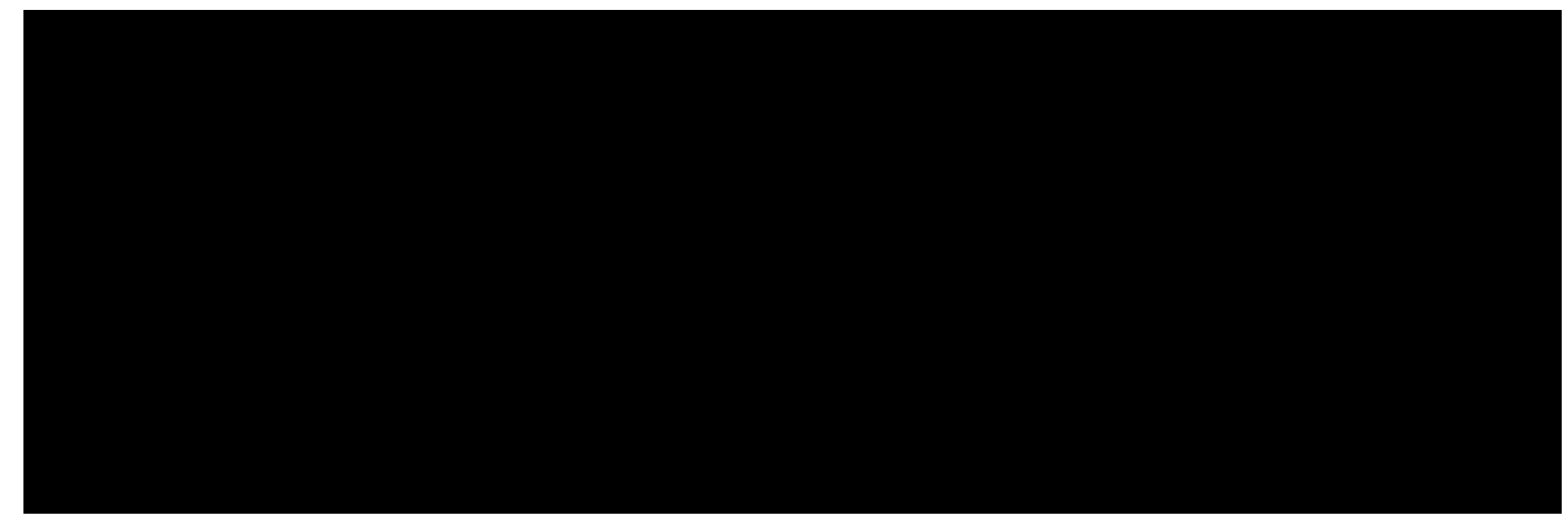

F. $=$ Fistula

Abdominal PSARP $+\mathrm{T}=$ Posterior sagital ano rectal plasty plus abdominal time

PARUVP $=$ Posterior ano rectal uretro vaginal plasty. This may be alone or plus descent of the urogenital sinus

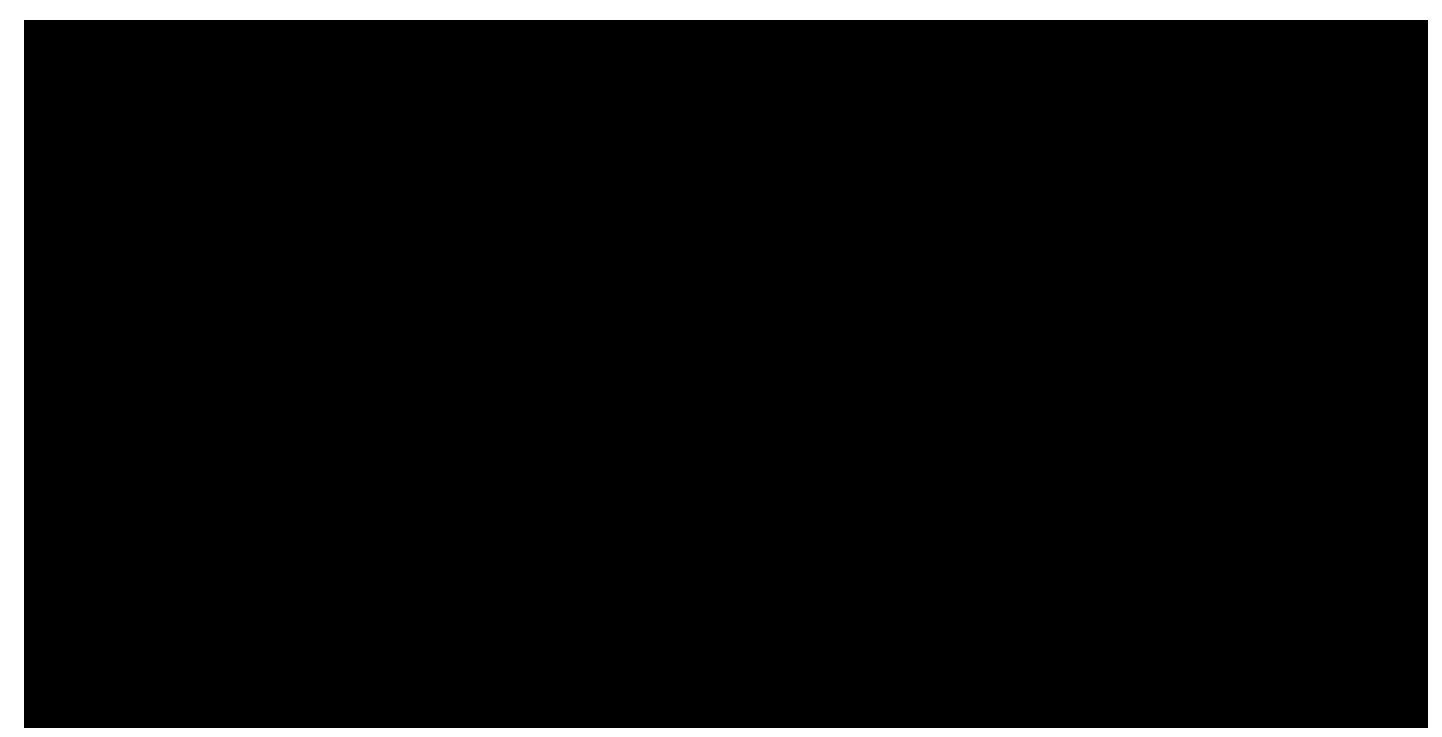

Acta méd. costarric. Vol 52 (2), April- June 2010 


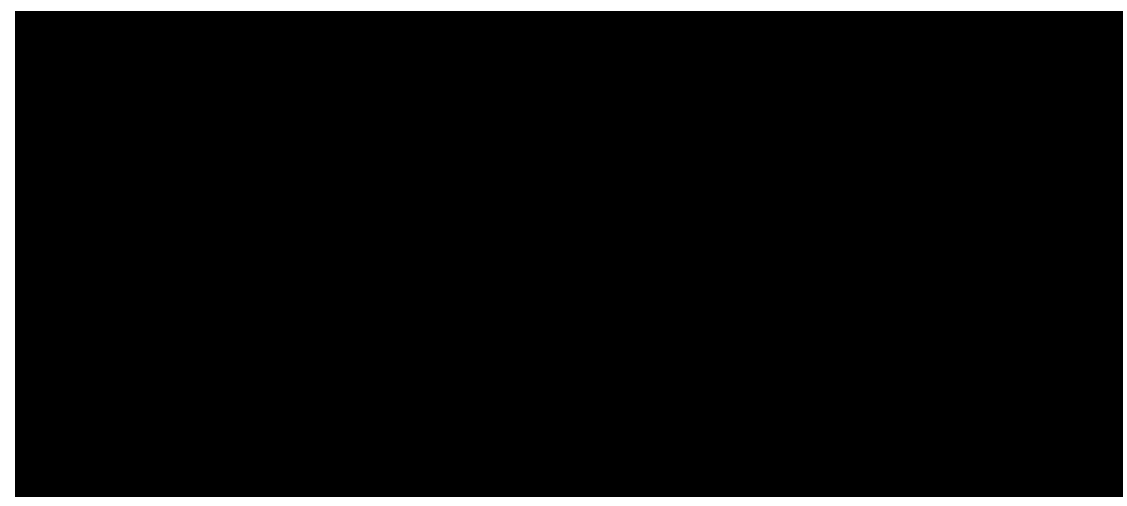

this procedure. The National Children's Hospital has begun using this technique, but such cases are not included in this article.

\section{Discussion}

\section{Fistula at the bladder neck}

Malformations with fistula at the bladder neck are difficult to treat. They are associated to a high percentage of sacral and neurological malformations, which influences the results. They are accessed posterior sagitally, they need a wide dissection which favors lesion of muscular and neural elements. It is necessary to perform a laparotomy to complete the descent, which could have caused the negative results obtained in this group, since $83.3 \%$ of the patients are incontinent. Similar data have been reported by Peña, with only $15 \%$ of the patients having a normal function.

This information motivated the search for other options for treatment, which led to Georgeson and Randolph ${ }^{4}$ to use the laparoscopic technique, avoiding the abdominal lesion and assuring a much clearer vision of the dissection of the fistula. Besides, the lesion to muscles and nerves is practically absent. Using the endoscopic vision, you can appreciate better the anus elevator muscle and the rectum that will descend may be placed between them. It is expected therefore, to obtain better results from
The distal colography with pressure demonstrates the fistula in only $50 \%$ of the patients, one of these cases documents that the location of the opening was at the prostatic urethra. It is important to improve the results of the test since this type of patient will be resolved using the laparascopic technique.

Out of the incontinent patients, only a $40 \%$ had a SI less than 0.3 , or sacral agenesia, a factor that regardless of the surgical technique, allows the findings of the negative results.

\section{Prostatic fistula}

The prostatic fistulas must be diagnosed before operation; therefore clinical data is very important, such as the contaminated urine with meconium in the newborn and the perineum without creases. However, the most important data is given by the distal colography with pressure. A careful bowel management of the distal segment after performing the colostomy to clean out all the meconium is very important to demonstrate the fistula. If this is not done correctly, the fistula will not be shown clearly.

Since it is a high lesion, the perineum area requires an extended and careful dissection, which may cause a lesion to the urethra, accident which was present in 2 cases. These patients

Acta méd. costarric. Vol 52 (2), April- June 2010 
have also been managed laparascopically and it is probable that with an increase in experience using this technique, they will be resolved this way in the future. This type of fistula was shown in most cases (75\%), probably because the technique was performed with more pressure or because the distal colostomy area had been cleaned better. Both patients that experienced an opening of the urethra during the operation were repaired and did not present further problems. There was also one case of inverted colostomy in this group.

The results of this group were not satisfactory: $50 \%$ are continent (constipated patients were classified as continent and the percentage is overall the evaluated cases, not including those who had not been evaluated because of their age). Half of the incontinent cases of this group had a SI greater than 0.3.

\section{Bulbar fistula}

Dissection is easier with the bulbar fistulas and generally homeostasis must be done carefully because the corpus spongiosum bulb is vascularized, however, these are better cases than the prostatic and the bladder cases. They have less bone malformations and better muscular complex; therefore the functional results will be superior. Among the revised cases, $64.2 \%$ are continent, a lesser value that that reported by Peña $(80.9 \%){ }^{6}$

\section{Vestibular fistulas}

These fistulas have a good muscular component and in most cases they are not associated to malformations. The inclusion criteria were with a colostomy as the first surgery, and later a PSARP and closure of the colostomy at a tertiary surgery. Some groups ${ }^{7}$ do a PSARP as a first surgery, without protecting Acta méd. costarric. Vol 52 (2), April- June 2010 themselves with a colostomy; out of the 5 of the patients treated with this procedure, one of them had a megasigmoid with led to a resection, and another case had to be intervened with PSARP due to a prolapsed anal mucosa.

Distal colography is not necessary in these patients since the diagnosis is made clinically only. The results are good: $85.3 \%$ continent (those evaluated and constipated were included) and $18 \%$ incontinent, out of which $66 \%$ present a SI less than 0.3 , or sacral agenesia, which caused negative results since before the treatment.

\section{Perineum fistulas}

Perineum malformations are the largest group, with the most cases. They have been resolved with a limited PSARP in the great majority, and a colostomy was used only when it was associated with another malformation that required an urgent treatment. (Esophageal A) They are the patients with the best functional results and only the incontinent patients have sacral malformations or very low sacral indices or neurological lesions, with a $10 \%$ of these cases.

In a $16.3 \%$ there were complications with the anal suture which caused another reoperation. This is a high percentage that must be improved.

Out of the 7 patients were a colostomy was done as a primary surgery, the decision was made for 4 of them based of their age, and the remaining 3 probably obeyed a negative initial diagnosis. Currently, bowel management is performed and intervened without a colostomy. In an $87.6 \%$, acceptable results were obtained since they are continent or constipated. Out of the $12.3 \%$ of the incontinent, 1 had sacral agenesia, and 4 had a SI less than 0.3 , which turns them into bad cases before the treatment started.

\section{Anal stenosis}


Just one case had a colostomy performed since it was diagnosed as Hirschprung disease, which later on was disregarded and the colostomy was closed and managed with dilations. All the patients were continent.

\section{High without fistula}

These are difficult patients where an extensive dissection must be performed, which permits the complications to be frequent: two cases had urethral ruptures, therefore it was necessary to be extremely careful during the dissection to not cause a lesion, since this is much easier if there is absence of a fistula. One of the patients underwent a PSARP as a primary surgery as the only treatment.

The colostomy is the operation indicated and the one that offers the best results and avoids such complications.

The results from this group have been the best, with a $92.8 \%$ of the patients being continent between those evaluated.

\section{Low without a fistula}

They do not have the urine contaminated with the meconium and as the high fistulas; these must be evaluated 24 hours after birth. The study that allows the diagnosis is a simple radiography taken as described. This study shows the distance between the mark at the anal pit and the sacral fornix, is less than $1 \mathrm{~cm}$. This allows them to be addressed through the perineum and better results are obtained. $100 \%$ of the cases are continent.

\section{High cloaca}

A cloaca is defined as the junction of the rectus, the vagina, the urethra, as seen in the classification used. To obtain a good control over the feces and the urine, and a normal sexual function, is one of the greatest challenges of children's surgeries that may only be obtained at centers that have a large volume of these patients. There are two types of lesions, were the common channel is greater than $3 \mathrm{~cm}$ and the low ones, which measure less than $3 \mathrm{~cm}$. The high ones are the ones that show difficulty in their treatment. The experience in this series is very small, in fact only 2 cases, therefore they will not be commented on with extension of their treatment; which is described in the reports by Hendre, Peña and Levitt. ${ }^{7-11}$ Both patients are incontinent.

\section{Low Cloaca}

Emphasis will be made only on the mistakes made at the initial management of these patients, which are: 1) To not discover and treat on time the hydrocolpos, which may achieve very large sizes and are necessary to drain, not doing this will cause obstruction of the ureters and produce hydronefrosis. $^{12,13}$ 2) Not leaving an adequate portion of the colon at the distal opening of the colostomy, since we must always assume that this portion of the colon could be needed for reconstruction of the vagina. 3) Not doing a good radiological evaluation, were a cystography should be done besides the colography. 4) Not doing a cystoscopy that allows us to measure the length of the common channel.

\section{Associated congenital malformations}

This is a very important subject in these patients and deserves an independent analysis, which is being worked on.

All the cases were studied in a complete manner to confirm or discard the diagnosis of some associated congenital disease, some which had to be managed immediately, as were the 8 cases of esophageal atresia and the case that had an omphalocele. The other patients were operated after, and in those urological cases, we tried to take advantage of those surgical spaces were laparotomy had to be performed to perform simultaneously a urological correction, with the 
objective to preserve renal function to its maximum. In most cases this was not possible and more time passed by than was advised for the correction of urological malformations. ${ }^{16}$

Vertebral malformations were analyzed carefully and in most cases, a sacral index was determined, in the same manner Peña does.

The frequent presentation of malformations forces the treatment and the control of these pathologies to be performed in a center that may resolve all these issues.

\section{Colostomy}

In the case of a patient with anorectal malformations, the first issue that must be resolved is whether or not a colostomy is necessary. In $55.5 \%$ of the patients, a colostomy was performed, for which it is considered fundamental to highlight the type as well as were the openings should be placed, and to pay special attention to not make the mistakes illustrated in Figure $3{ }^{17}$

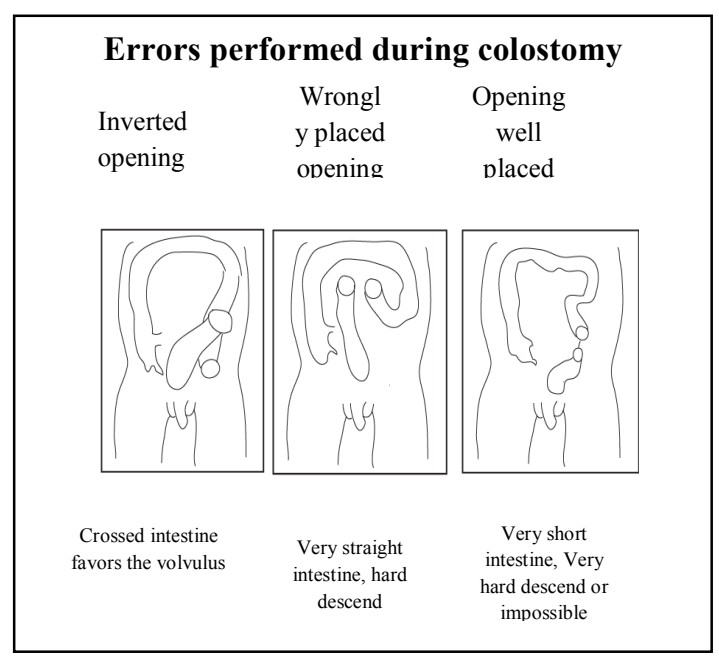

Figure 2

The colostomy should always be done with two separate openings, to avoid urinary infections and to be able to maintain the terminal portion of the rectum clean of meconium. The distal opening will be managed as a fistula.

Acta méd. costarric. Vol 52 (2), April- June 2010
The placement of the colostomy should be at the beginning of the sigmoid colon, leaving a large portion of this to be able to ease the descent in the correction of the anorectal malformation, as shown in Figure 3, were a colography has been placed that measures the distance of the colon. This is done in a normal manner and has been of great utility.

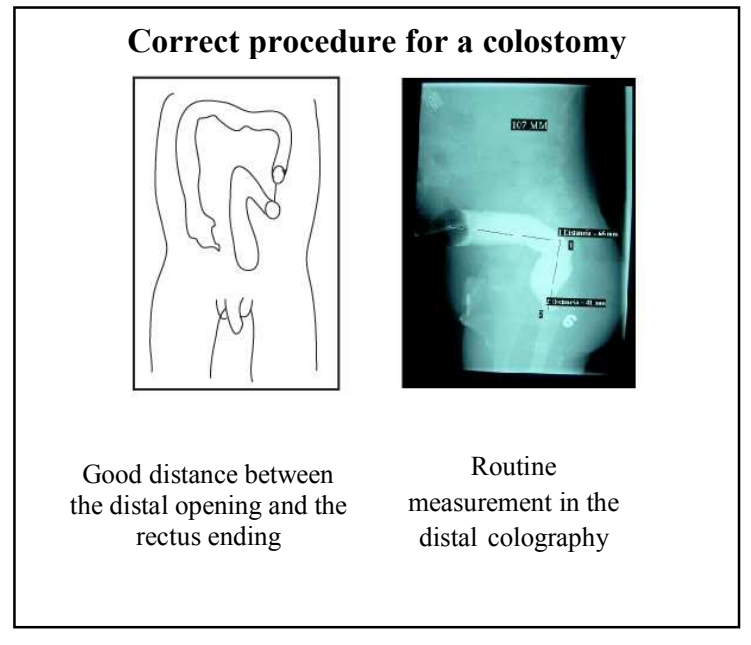

Conclusions

The children born with an anorectal malformation are complex patients, who deserve an integrated study and prioritized programming of the multiple interventions needed for their treatment. The grouping, according to the classification used, allows a better analysis and interpretation of the results.

The 10 year experience is shown in the management of these malformations at the center and guidelines are given for the diagnosis and treatment, which may be useful, such as: carefully cleaning the distal segment of the colostomy and placing pressure over the distal colography to show the fistula; same as the recommendations offered in relation to the 
colostomy. It is expected that in the future, changes in treatment will be proposed, as product of the advances in the diagnosis and surgical techniques. These patients must be controlled in a specific consult for a long time and procedures that allow evaluation and treatment of the constipation and incontinence must be used, in order to have a normal life, which is possible.

\section{References}

1 DeVries PA, Pena A. Posterior sagittal anorectoplasty. J Pediatr Surg. Oct 1982;17:63843.

2 Peña A Anorectal malformations. Semin Pediatr Surg. 1995:4910:35-45.

3 Beals RK, Robbins JR, Rolfe B. Anomalies associated with vertebral malformations. Spine. 1993;18:1329-32

4- Keith E. Georgeson, Thomas H. Inge and Craig T. Albanese. Laparoscopically assisted anorectal pull-through for high imperforate anus-A new technique. J Pediatric Surg. 2000; 35: 927-93.

5-Gross GW, Wolfson PJ, Peña A. Augmentedpressure colostogram in imperforate anus with fistula. Pediatr Radiol 1991;21:560-2.

6- Pena A. Management of anorectal malformations during the newborn period. World J Surg. 1993;17:385-92.
7- Hendren WH. Cloaca, the most severe degree of imperforate anus: experience with 195 cases. Ann Surg. 1998;228:331-46.

8- Hendren WH. Cloacal malformations: experience with 105 cases. J Pediatr Surg. 1992; 27:890-901.

9- Hendren WH. Pediatric rectal and perineal problems. Pediatr Clin North Am. 1998; 45:1353-72.

10- Levitt MA, Stein DM, Pena A. Gynecologic concerns in the treatment of teenagers with cloaca. J Pediatr Surg. 1998;33:188-93.

11- Levitt MA, Pena A. Pitfalls in the management of newborn cloacas. Pediatr Surg Int. $2005 ; 21: 264-9$.

12- Levitt MA, Stein DM, Pena A. Gynecologic concerns in the treatment of teenagers with cloaca. J Pediatr Surg. 1998; 33:188-93.

13- Rich MA, Brock WA, Pena A. Spectrum of genitourinary malformations in patients with imperforate anus. Pediatric Surg Intl. 1988; 3:110-113.

16- Tovilla-Mercado J M, Peña A. Acta Pediatr Mex. 2008;29:147-50.

17 Pena A, Migotto-Krieger M, Levitt MA. Colostomy in anorectal malformations: a procedure with serious but preventable complications. J Pediatr Surg. 2006; 41:748-56.

Translated by: María Fernanda Valverde 